\title{
Optimization of Extraction Process of Methyl Eugenol and Asarinin in Asarum with Deep Eutectic Solvent Based on the Response Surface Methodology
}

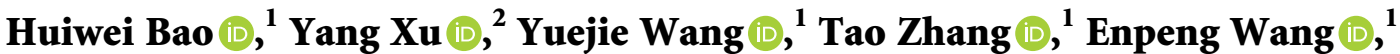 \\ Xin Huang $\left({ }^{1},{ }^{1}\right.$ Changbao Chen $\mathbb{}^{1},{ }^{1}$ and Fangxin Liu $\mathbb{\circledR}^{1}$ \\ ${ }^{1}$ College of Pharmacy, Changchun University of Chinese Medicine, Changchun 130117, China \\ ${ }^{2}$ College of Pharmacy, Baicheng Medical College, Baicheng 137000, China \\ Correspondence should be addressed to Changbao Chen; ccb2021@126.com and Fangxin Liu; 1136244403@qq.com
}

Received 7 August 2021; Accepted 28 August 2021; Published 18 September 2021

Academic Editor: Liviu Mitu

Copyright (c) 2021 Huiwei Bao et al. This is an open access article distributed under the Creative Commons Attribution License, which permits unrestricted use, distribution, and reproduction in any medium, provided the original work is properly cited.

\begin{abstract}
To explore a green and efficient extraction technology for the extraction of active ingredients of Asarum, the deep eutectic solvent combined with ultrasonic was applied to compare the extraction efficiency of 10 kinds of deep eutectic solvents, taking the extraction rate of methyl eugenol and asarinin as indices. Single-factor experiments were adopted to investigate the influence of molar ratio, liquid-to-solid ratio, eddy time, ultrasonic time, and temperature of the deep eutectic solvent on the extraction rate of methyl eugenol and asarinin. Based on single-factor experiments, the surface response methodology was used to optimize the extraction process conditions. The results showed that the optimum extracting process conditions of methyl eugenol and asarinin in Asarum consisted of a ratio of choline chloride to glycerol of $1: 3$, a DES volume of $2 \mathrm{~mL}$, an ultrasonic temperature of $60^{\circ} \mathrm{C}$, an ultrasonic time of $30 \mathrm{~min}$, and a vortex oscillation of $7 \mathrm{~min}$. Under the optimum extracting process conditions, the contents of methyl eugenol and asarinin were $1.9428 \mathrm{mg} / \mathrm{g}$ and $0.9989 \mathrm{mg} / \mathrm{g}$, respectively, and the comprehensive index was 2.3280 (RSD of $1.91 \%)$. The results were close to the predicted values of the response surface model, demonstrating the applicability of the model. The extraction rate of methyl eugenol and asarinin in Asarum by this method was higher than that of water extraction and alcohol extraction, which fully indicated the high efficiency of ultrasonic-assisted green deep eutectic solvent extraction technology. The results provide data support for further development and utilization of Asarum.
\end{abstract}

\section{Introduction}

Asarums are the dry roots and rhizomes of Aristolochiaceae plants such as Asarum heterotropoides Fr. Schmidt var. mandshuricum (Maxim.) Kitag., Asarum sieboldii Miq. Var. seoulense Nakai, or Asarum sieboldii Miq. [1]. Asarum is pungent, warm in nature, and enters the heart, lung, and kidney meridians, with slight toxicity [2]. It has the effect of reliving the exterior syndrome and dispersing cold, dispelling wind and relieving pain and opening into five organs, and warming the lung and resolving fluid [3]. Asarum contains a variety of components, including volatile oil, lignans, flavonoids, steroids, polysaccharides [4-7], asarinin, methyl eugenol, safrole, and so on [8]. Asarum is commonly used in the clinical treatment of common cold due to wind cold, headache, toothache, stuffy and runny nose, allergic rhinitis, nasosinusitis, rheumatism and pain syndrome, and phlegm and retained fluid, dyspnea with cough [9].

In 2003, Abbott et al. [10] first found a solvent composed of hydrogen bond acceptors and donors with certain molar ratio and called it deep eutectic solvent (DES). As it has good physical and chemical properties, such as green, low melting point, easy to synthesis, low cost, strong solubility, and can be repeatedly used $[11,12]$, DESs currently have gradually replaced organic solvents and ionic liquids and showed great potential in the extraction and separation of natural plants [13-15]. In recent years, water-soluble DESs based on 
choline chloride have been widely applied in various fields, from physical, material, and chemical to the efficient extraction and separation of alkaloids, flavonoids, polysaccharides, and acids from medicinal plants $[16,17]$.

In the study, 10 kinds of DESs were first used in the extraction of the active ingredients of Asarum. With the index of the extraction rate of methyl eugenol and asarinin, the response surface methodology was applied to analyze the optimal conditions of the extraction of methyl eugenol and asarinin from Asarum, which was subsequently compared with the traditional extraction method, so as to provide a more green, efficient, and economical novel extraction method, promote the deep development of Asarum, and provide a theoretical basis for the comprehensive development and utilization of Asarum.

\section{Materials and Methods}

2.1. Reagents. Asarum used in this experiment was from Sichuan Province in China and purchased from Jilin Pharmacy (Changchun), and all of them met the requirements of Chinese Pharmacopoeia (2020 edition). Chromatography-grade methanol (batch no. 20200601) and acetonitrile (batch no. 20200601) were purchased from Fisher Company, USA; analytical grade phosphoric acid (batch no. 20170408) was obtained from Tianjin Guangfu Science and Technology Development Co., Ltd.; ultrapure water was acquired from Hangzhou Wahaha Co., Ltd.; methyl eugenol (batch no. 111642-200301) and asarinin (batch no. 111889-201705) were acquired from the National Institute of Food and Drug Control; choline chloride (batch no. 150120) was obtained from Shanghai Zhanyun Chemical Co., Ltd.; maltose (batch no. 117101), malic acid (batch no. 117101), lactic acid (batch no. 117101), fructose (batch no. 117001), xylitol (batch no. 117101), and citric acid (batch no. 117101) were acquired from Zhengzhou Kangyuan Chemical Products Co., Ltd.; phenol (batch no. 170902) and acetic acid (batch no. 117101) were purchased from Xilong Scientific Co., Ltd.; glycerin (batch no. 20180406) was purchased from Guangzhou Yaya Cosmetics Co., Ltd.; propylene glycol (batch no. 20180309) was obtained from Tianjin Zhonghe Shengtai Chemical Co., Ltd.; and urea (batch no. 20170109) was purchased from Tianjin Ruijinte Chemical Co., Ltd.

2.2. Instrumentation. The chromatographic analysis was performed with the use of Huapu S3000 high performance liquid chromatography system (including four-element low-pressure stirring pump, automatic sampler, column box, 1100 diode array detector, and chemical workstation). Jp-300 G ultrasonic extractor was purchased from Wuhan Jiapeng Electronics Co., Ltd. AB135-S electronic balances were purchased from Mettler Toledo International Ltd. Q1-901 vortex instrument was purchased from Haimen Qilin Beier instrument Manufacturing Co., Ltd. TGL-16 refrigerated centrifuge was purchased from Hunan Xiangyi Laboratory Instrument Development Co., Ltd.
2.3. HPLC Method for the Determination of Asarum. The chromatographic column was Diamonsi ${ }^{\oplus} \mathrm{C}_{18}(4.6 \times 250 \mathrm{~mm}$, $5 \mu \mathrm{m})$. The mobile phase included acetonitrile- $0.1 \%$ phosphoric acid aqueous solution. The selected wavelength was $287 \mathrm{~nm}$, the injection volume was $10 \mu \mathrm{L}$, and the column temperature was $40^{\circ} \mathrm{C}$. The detailed gradient elution conditions are displayed in Table 1. HPLC chromatogram of Asarum is given in Figure 1.

2.4. Preparation of DESs. In this paper, choline chloride was selected as the hydrogen bond acceptor (HBA) and the 10 materials in Table 2 were selected as the hydrogen bond donors (HBD) to prepare DESs considering their nature, safety, and low cost. HBA and HBD were accurately weighed and mixed at a certain molar ratio, heated, and stirred at $80-100^{\circ} \mathrm{C}$ until a clear and uniform liquid was formed.

2.5. Extraction of Methyl Eugenol and Asarinin from Asarum. The process of preparation of the test product is shown in Figure $2.50 \mathrm{mg}$ of Asarum powder was weighed and subsequently added to the DES at a solid-liquid ratio of $1: 30(\mathrm{~g} /$ $\mathrm{mL}$ ), warm bath for $5 \mathrm{~min}$, eddy oscillation for $5 \mathrm{~min}$, ultrasonic extraction at $60^{\circ} \mathrm{C}$ for $30 \mathrm{~min}$, ultrasonic power of $200 \mathrm{~W}$ [18], eddy oscillation for $1 \mathrm{~min}$, and centrifugation at $3000 \mathrm{r} / \mathrm{min}$ for $5 \mathrm{~min}$ at obtain the Asarum extract.

2.6. Box-Behnken Response Surface Experimental Design. According to the experimental design principles of Box-Behnken and the results of the previous single-factor pretest, the most influencing factors of the extraction of Asarum (molar ratio, DES volume, ultrasonic temperature $\left({ }^{\circ} \mathrm{C}\right)$, and ultrasonic time $(\mathrm{min})$ ) were selected and numbered $-1,0$, and 1 , respectively. The extraction rate of methyl eugenol and asarinin was averaged to obtain the comprehensive index (CI) as the investigation index. 29 test sites were designed for the response surface test, and then the optimal process conditions were verified. The test factors and level design are shown in Table 3.

\section{Results and Discussion}

\subsection{Method Validation}

3.1.1. Linear Range and Detection Limit. Prepared standard series solutions were injected under the established chromatographic conditions to obtain HPLC peak areas at different concentrations. There was a linear relationship between peak area and the concentrations of methyl eugenol and asarinin in the range of $0.005-0.495 \mathrm{mg} / \mathrm{mL}$ and $0.002-0.230 \mathrm{mg} / \mathrm{mL}$, respectively. The results are shown in Table 4.

3.1.2. Precision and Accuracy. The precision and accuracy of the analysis method were verified by the precision test, repeatability test, and sampling recovery test. The same Asarum extract was continuously injected 6 times according to the above HPLC analysis conditions, and the RSDs of methyl eugenol and asarinin peak area were $0.54 \%$ and 
TABLE 1: Gradient elution conditions.

\begin{tabular}{lccr}
\hline Time $(\mathrm{min})$ & Flow rate $\left(\mathrm{mL} \cdot \mathrm{min}^{-1}\right)$ & A (\%) & \\
\hline 0 & 1.5 & 38 & B (\%) \\
13 & 1.5 & 38 & 62 \\
13.1 & 1.0 & 43 & 57 \\
27 & 1.0 & 43 & 57 \\
28 & 1.0 & 51 & 49 \\
45 & 1.0 & 51 & 49 \\
\hline
\end{tabular}

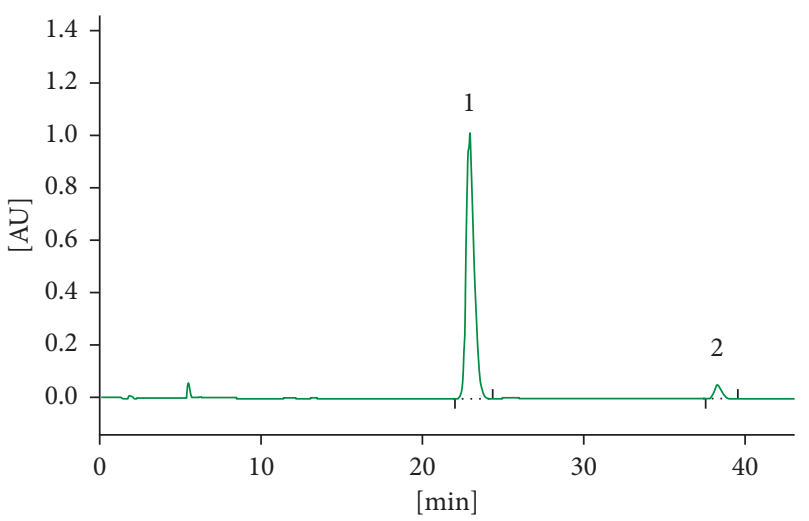

(a)

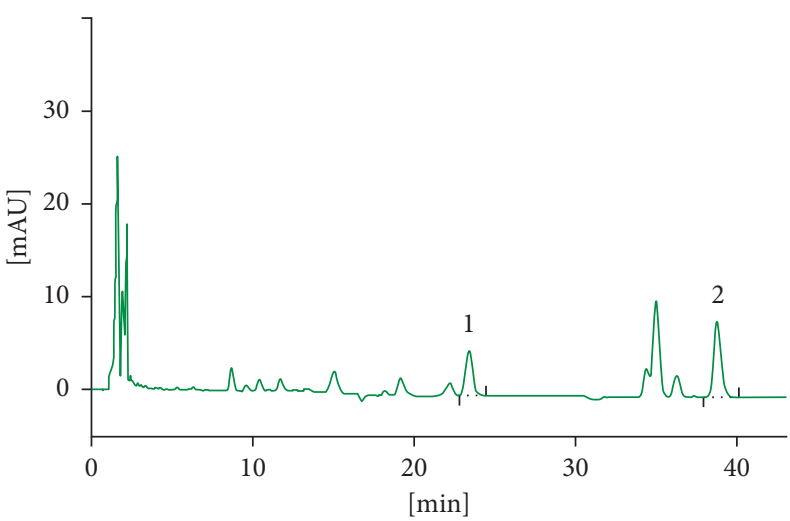

(b)

Figure 1: HPLC chromatogram of Asarum. (a) Reference substance and (b) test substance (1, methyl eugenol; 2, asarinin).

TABLE 2: Different types of DESs.

\begin{tabular}{llcr}
\hline No. & HBA & HBD & Molar ratio \\
\hline 1 & Choline chloride & Maltose & $1: 1$ \\
2 & Choline chloride & Malic acid & $1: 1$ \\
3 & Choline chloride & Lactic acid & $1: 2$ \\
4 & Choline chloride & Fructose & $1: 1$ \\
5 & Choline chloride & Glycerol & $1: 2$ \\
6 & Choline chloride & Propylene glycol & $1: 2$ \\
7 & Choline chloride & Xylitol & $1: 1$ \\
8 & Choline chloride & Urea & $1: 2$ \\
9 & Choline chloride & Acetic acid & $1: 2$ \\
10 & Choline chloride & Citric acid & $1: 2$ \\
\hline
\end{tabular}

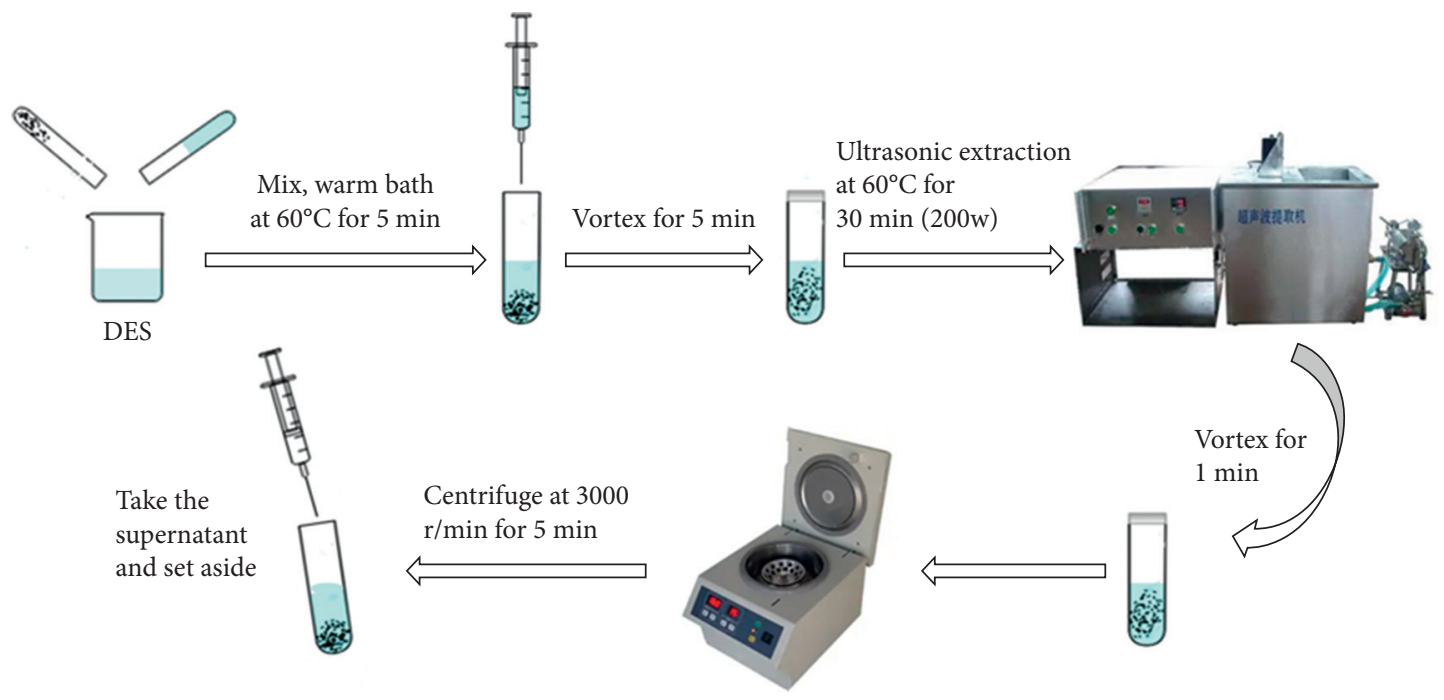

FIgURE 2: Preparation diagram of test product. 
TABLE 3: Test factors and level design of response surface methodology.

\begin{tabular}{lccc}
\hline Factors & \multicolumn{3}{c}{ Level } \\
& -1 & 0 & 1 \\
\hline A. Molar ratio & $1: 2$ & $1: 3$ & $1: 4$ \\
B. DES volume $(\mathrm{mL})$ & 1 & 1.5 & 2 \\
C. Ultrasonic temperature $\left({ }^{\circ} \mathrm{C}\right)$ & 50 & 60 & 70 \\
D. Ultrasonic time (min) & 20 & 30 & 40 \\
\hline
\end{tabular}

TABLE 4: Linear range and detection limit.

\begin{tabular}{lccc}
\hline Constituent & Concentration range $(\mathrm{mg} / \mathrm{g})$ & Linearity curve & $r$ \\
\hline Methyl eugenol & $0.005-0.495$ & $y=6281.8 x-13.595$ & 0.9998 \\
Asarinin & $0.002-0.230$ & $y=22335 x-27.887$ & 0.9998 \\
\hline
\end{tabular}

$0.78 \%$, respectively. 6 Asarum extracts were prepared and analyzed by HPLC using the above method. The average contents of methyl eugenol and asarinin were $1.528 \mathrm{mg} / \mathrm{g}$ and $0.802 \mathrm{mg} / \mathrm{g}$, and the RSDs were $1.06 \%$ and $1.72 \%$, respectively.

Six pieces of Asarum with known content were taken, each of which was about $25 \mathrm{mg}$. $0.2 \mathrm{~mL}$ of methyl eugenol (at a concentration of $0.468 \mathrm{mg} / \mathrm{mL}$ ) and $0.1 \mathrm{~mL}$ of asarinin (at a concentration of $0.453 \mathrm{mg} / \mathrm{mL}$ ) were accurately transferred to a centrifugal tube. After the solvent was dried in a water bath, the measured Asarum powder was added. Asarum extract was extracted according to the method in Section 2.3 and analyzed by HPLC. The average recovery percentages of methyl eugenol and asarinin were $99.07 \%$ and $98.89 \%$, respectively, with RSDs of $1.90 \%$ and $1.19 \%$. It means that this method was of high precision and accuracy.

3.2. Effect of DES Types. In this paper, choline chloride is used as the HBA, which can combine with hydrogen bond donors to form hydrogen bond, and it has the advantages of low cost, low toxicity, and biodegradation [19]. The extraction of methyl eugenol and asarinin is the most important factor that should be considered in the optimization of the HBD component. In this study, the effects of 10 kinds of DESs (see Table 1) on the extraction amount of methyl eugenol and asarinin were investigated. The results are shown in Figure 3. DES-5, the combination of choline chloride with glycerol, had the highest efficiency in the extraction of methyl eugenol and asarinin from Asarum.

3.3. Effect of Molar Ratio. Because the recoveries of different molar ratios about DES may have different extraction properties, several DESs with different molar ratios were synthesized for the extraction of methyl eugenol and asarinin, as it is a standard to evaluate the extracting abilities of different DESs. In this paper, the effects of DES at the molar ratios of choline chloride to glycerol of $1: 1,1: 2,1: 3$, and 1 : 4 on the extraction rates of methyl eugenol and asarinin from Asarum were investigated. As shown in Figure 4, when the molar ratio of choline chloride to glycerol is $1: 3$, the extraction efficiency is the highest, so this molar ratio is selected for subsequent experiments.
3.4. Effect of DES Volume. As the only extraction solvent, the amount of DES directly affects the extraction efficiency of methyl eugenol and asarinin. The main reason is that if the amount of extraction solvent is insufficient, the target components cannot be completely extracted, while excessive DES will lead to a waste. Therefore, this paper focuses on the DES volume in the range of $0.5-2.0 \mathrm{~mL}$. As shown in Figure 5, when the DES volume was $1.5 \mathrm{~mL}$, the extraction rates of methyl eugenol and asarinin were the highest. Finally, $1.5 \mathrm{ml}$ of DES was chosen as the optimum amount of extraction solvent in this study.

3.5. Effect of Ultrasonic Temperature. In the whole extraction process, temperature has a significant effect on the extraction efficiency of the target compounds. Generally, an increased temperature will accelerate the movement and transfer of the materials [20]. In this paper, the effect of temperature in the range of $40-70^{\circ} \mathrm{C}$ on the extraction rate of methyl eugenol and asarinin was investigated. As can be seen from Figure 6, at the temperature of $40-60 \mathrm{C}$, the extraction rate of methyl eugenol and asarinin increased, but when the temperature is higher than $60^{\circ} \mathrm{C}$, the extraction rate of both of them decreased, which may be caused by the destruction of the original structure of some substances at excessively high temperature. Therefore, $60^{\circ} \mathrm{C}$ was set as the optimal condition in the following study.

3.6. Effect of Ultrasonic Time. Ultrasonic time is also one of the important factors affecting the extraction effect. As can be seen from Figure 7, the ultrasonic time in the range of 10-30 min was positively correlated with the extraction rate of methyl eugenol and asarinin, but after $30 \mathrm{~min}$, the ultrasonic time was negatively correlated with the extraction rate. This may be because the longer the time, the greater the energy generated by the ultrasonic wave, the stronger the cavitation effect formed in the solution, the stronger the damage to the cell wall of Asarum, and the higher the extraction yield. However, as the extraction time increases, the temperature also increases gradually, and the system is under the action of ultrasonic radiation and thermal effect at the same time, which may lead to double degradation $[21,22]$. 


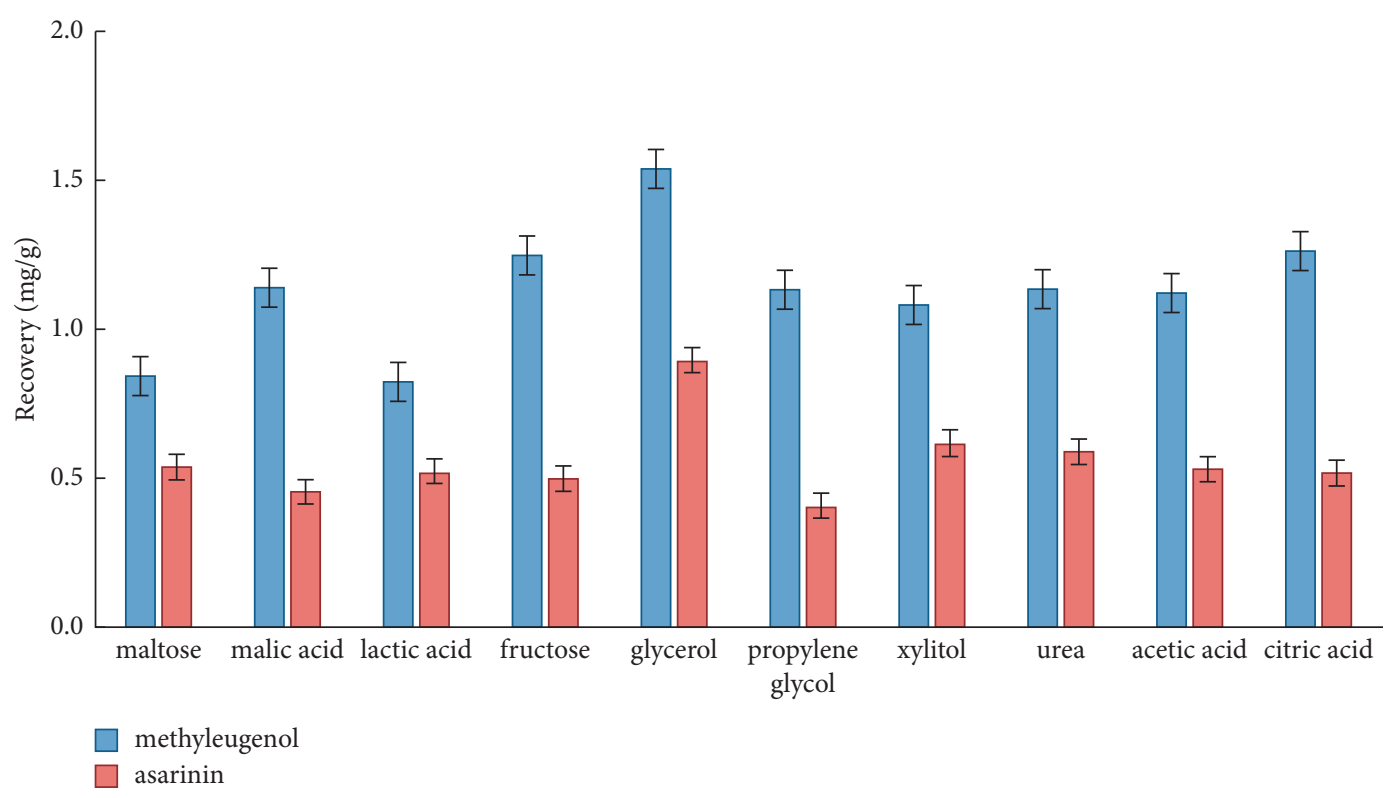

FIGURE 3: Effect of DES types, extraction conditions: sample amount, $50 \mathrm{mg}$; DES volume, $1.5 \mathrm{~mL}$; ultrasonic temperature, $60^{\circ} \mathrm{C}$; ultrasonic time, $30 \mathrm{~min}$; and vortex oscillation, $5 \mathrm{~min}$.

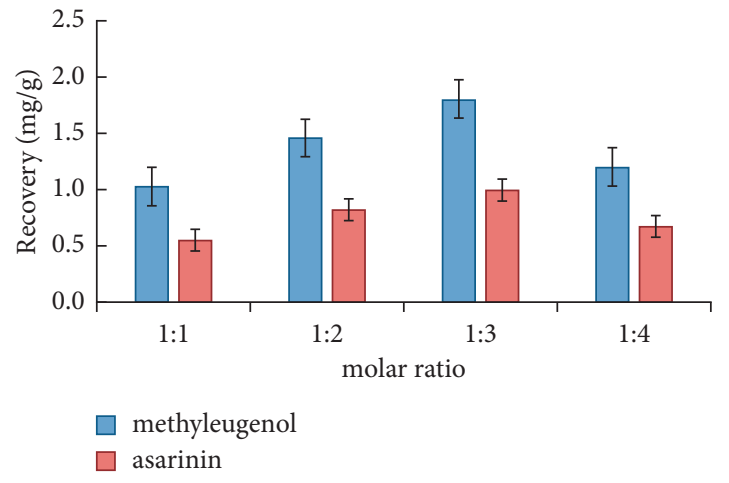

FIGURE 4: Effect of molar ratio, extraction conditions: sample amount, $50 \mathrm{mg}$; DES volume, $1.5 \mathrm{~mL}$; ultrasonic temperature, $60^{\circ} \mathrm{C}$; ultrasonic time, $30 \mathrm{~min}$; and vortex oscillation, $5 \mathrm{~min}$.

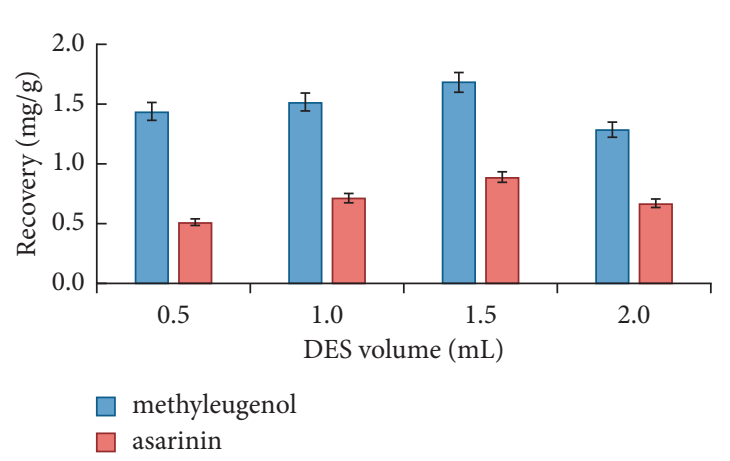

FIGURE 5: Effect of DES volume, extraction conditions: sample amount, $50 \mathrm{mg}$; molar ratio, $1: 2$; ultrasonic temperature, $60^{\circ} \mathrm{C}$; ultrasonic time, $30 \mathrm{~min}$; and vortex oscillation, $5 \mathrm{~min}$.

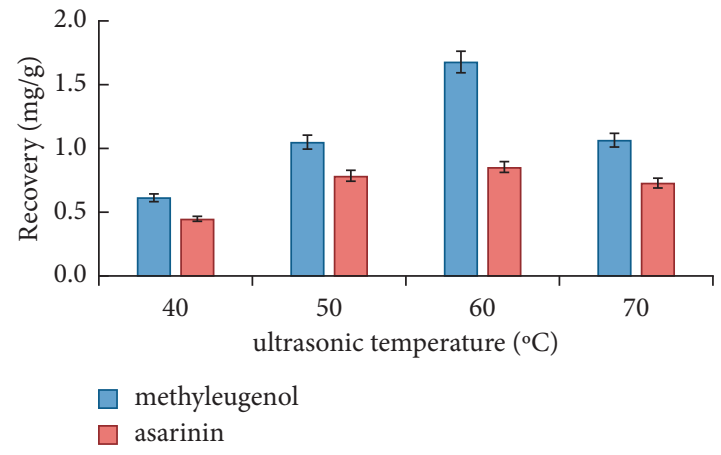

FIGURE 6: Effect of ultrasonic temperature, extraction conditions: sample amount, $50 \mathrm{mg}$; molar ratio, $1: 2$; DES volume, $1.5 \mathrm{~mL}$; ultrasonic time, $30 \mathrm{~min}$; and vortex oscillation, $5 \mathrm{~min}$.

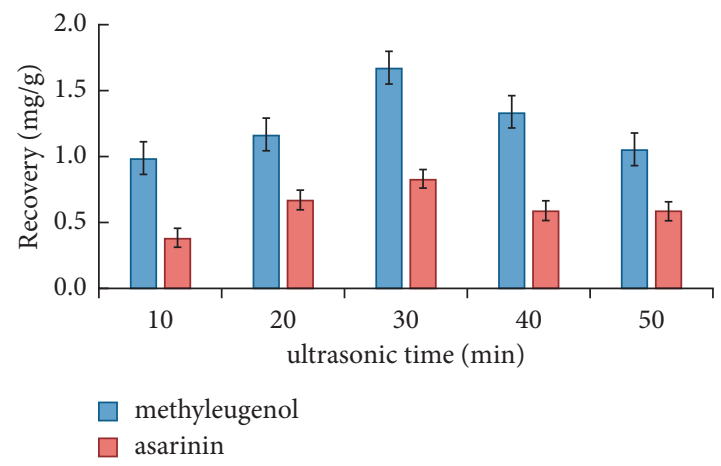

FIgURE 7: Effect of ultrasonic time, extraction conditions: sample amount, $50 \mathrm{mg}$; molar ratio, $1: 2$; DES volume, $1.5 \mathrm{~mL}$; ultrasonic temperature, $60^{\circ} \mathrm{C}$; and vortex oscillation, $5 \mathrm{~min}$. 


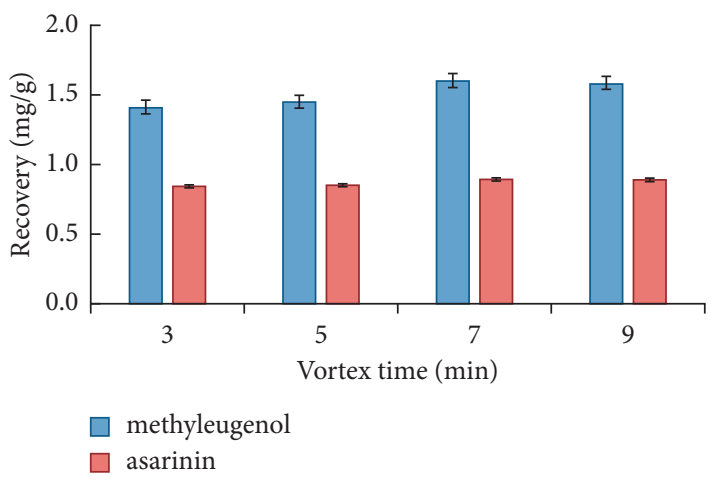

FIgUre 8: Effect of ultrasonic time, extraction conditions: sample amount, $50 \mathrm{mg}$; molar ratio, $1: 2$; DES volume, $1.5 \mathrm{~mL}$; ultrasonic temperature, $60^{\circ} \mathrm{C}$; and ultrasonic time, $30 \mathrm{~min}$.

TABLE 5: Analysis of variance of quadratic regression equation of Box-Behnken response surface.

\begin{tabular}{|c|c|c|c|c|c|c|c|c|c|c|c|}
\hline No. & A & $\mathrm{B}$ & $\mathrm{C}$ & $\mathrm{D}$ & $\mathrm{CI}$ & No. & $\mathrm{A}$ & B & $\mathrm{C}$ & $\mathrm{D}$ & CI \\
\hline 1 & 0 & -1 & 0 & 1 & 2.1642 & 16 & 1 & 0 & -1 & 0 & 2.0186 \\
\hline 2 & 0 & 0 & 0 & 0 & 2.2457 & 17 & -1 & -1 & 0 & 0 & 1.7488 \\
\hline 3 & 0 & 0 & 0 & 0 & 2.2293 & 18 & -1 & 0 & 0 & 1 & 1.4969 \\
\hline 4 & 0 & 1 & -1 & 0 & 2.1051 & 19 & -1 & 0 & 1 & 0 & 1.4810 \\
\hline 5 & 1 & 0 & 0 & -1 & 1.8751 & 20 & 1 & 0 & 1 & 0 & 1.8567 \\
\hline 6 & 0 & 0 & -1 & -1 & 2.0394 & 21 & 0 & 0 & 0 & 0 & 2.3719 \\
\hline 7 & -1 & 0 & 0 & -1 & 1.4474 & 22 & 0 & 0 & 1 & -1 & 2.2232 \\
\hline 8 & 1 & 0 & 0 & 1 & 1.8097 & 23 & 0 & 1 & 0 & 1 & 2.1923 \\
\hline 9 & 0 & 0 & 0 & 0 & 2.2433 & 24 & 0 & 0 & 1 & 1 & 2.3243 \\
\hline 10 & 0 & 1 & 1 & 0 & 2.3297 & 25 & 0 & -1 & 1 & 0 & 2.1863 \\
\hline 11 & 0 & 0 & 0 & 0 & 2.1759 & 26 & 0 & -1 & 0 & -1 & 2.1089 \\
\hline 12 & 0 & -1 & -1 & 0 & 2.0929 & 27 & -1 & 1 & 0 & 0 & 1.5489 \\
\hline 13 & 0 & 1 & 0 & -1 & 2.2702 & 28 & 0 & 0 & -1 & 1 & 2.2659 \\
\hline 14 & 1 & -1 & 0 & 0 & 1.5586 & 29 & -1 & 0 & -1 & 0 & 1.5348 \\
\hline 15 & 1 & 1 & 0 & 0 & 2.0212 & - & - & - & - & - & - \\
\hline
\end{tabular}

TABLE 6: Analysis of variance of the regression model.

\begin{tabular}{lccccc}
\hline Source & Sum of squares & Df & Mean square & $F$ value & $P$ value \\
\hline Model & 2.32 & 14 & 0.17 & 16.50 & $<0.0001$ \\
A. Molar ratio & 0.30 & 1 & 0.30 & 29.41 & $<0.0001$ \\
B. DES volume & 0.031 & 1 & 0.031 & 3.07 & 0.1018 \\
C. Ultrasonic temperature & $9.883 \mathrm{E}-003$ & 1 & $9.883 \mathrm{E}-003$ & 0.98 & 0.3379 \\
D. Ultrasonic time & $6.961 \mathrm{E}-003$ & 1 & $6.961 \mathrm{E}-003$ & 0.69 & 0.4190 \\
AB & 0.11 & 1 & 0.11 & 10.93 & 0.0052 \\
AC & $2.923 \mathrm{E}-003$ & 1 & $2.923 \mathrm{E}-003$ & 0.29 & 0.5980 \\
AD & $3.300 \mathrm{E}-003$ & 1 & $3.300 \mathrm{E}-003$ & 0.33 & 0.5755 \\
BC & $4.308 \mathrm{E}-003$ & 1 & $4.308 \mathrm{E}-003$ & 0.43 & 0.5230 \\
BD & $4.436 \mathrm{E}-003$ & 1 & $4.436 \mathrm{E}-003$ & 0.44 & 0.5170 \\
CD & $3.930 \mathrm{E}-003$ & 1 & $3.930 \mathrm{E}-003$ & 0.39 & 0.5416 \\
A2 & 1.77 & 1 & 1.77 & 176.57 & $<0.0001$ \\
B2 & $6.459 \mathrm{E}-003$ & 1 & $6.459 \mathrm{E}-003$ & 0.64 & 0.4359 \\
C2 & $1.500 \mathrm{E}-003$ & 1 & $1.500 \mathrm{E}-003$ & 0.15 & 0.7049 \\
D2 & 0.013 & 1 & 0.013 & 1.32 & 0.2692 \\
Residual & 0.14 & 14 & 0.010 & & Significant \\
\hline Lack of fit & 0.12 & 10 & 0.012 & 2.30 & 0.2187 \\
Pure error & 0.021 & 4 & $5.199 \mathrm{E}-003$ & & \\
Cor. total & 2.46 & 28 & & & \\
R2 & 0.9429 & & & & Not significant \\
R2Adj & 0.8857 & 5.01 & & & \\
CV (\%) & 12.372 & & & & \\
Adeq precision & & & & & \\
\hline
\end{tabular}



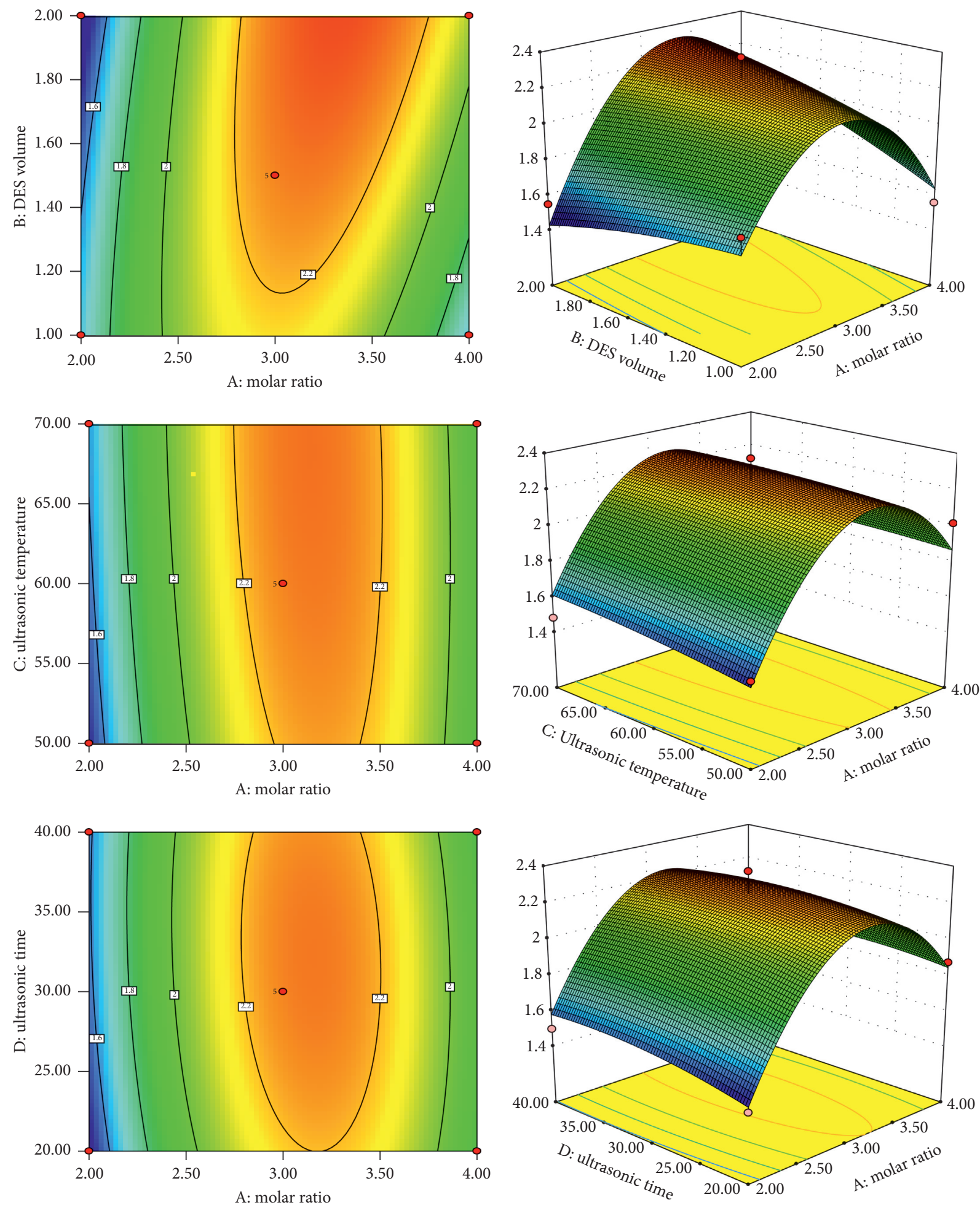

(a)

Figure 9: Continued. 

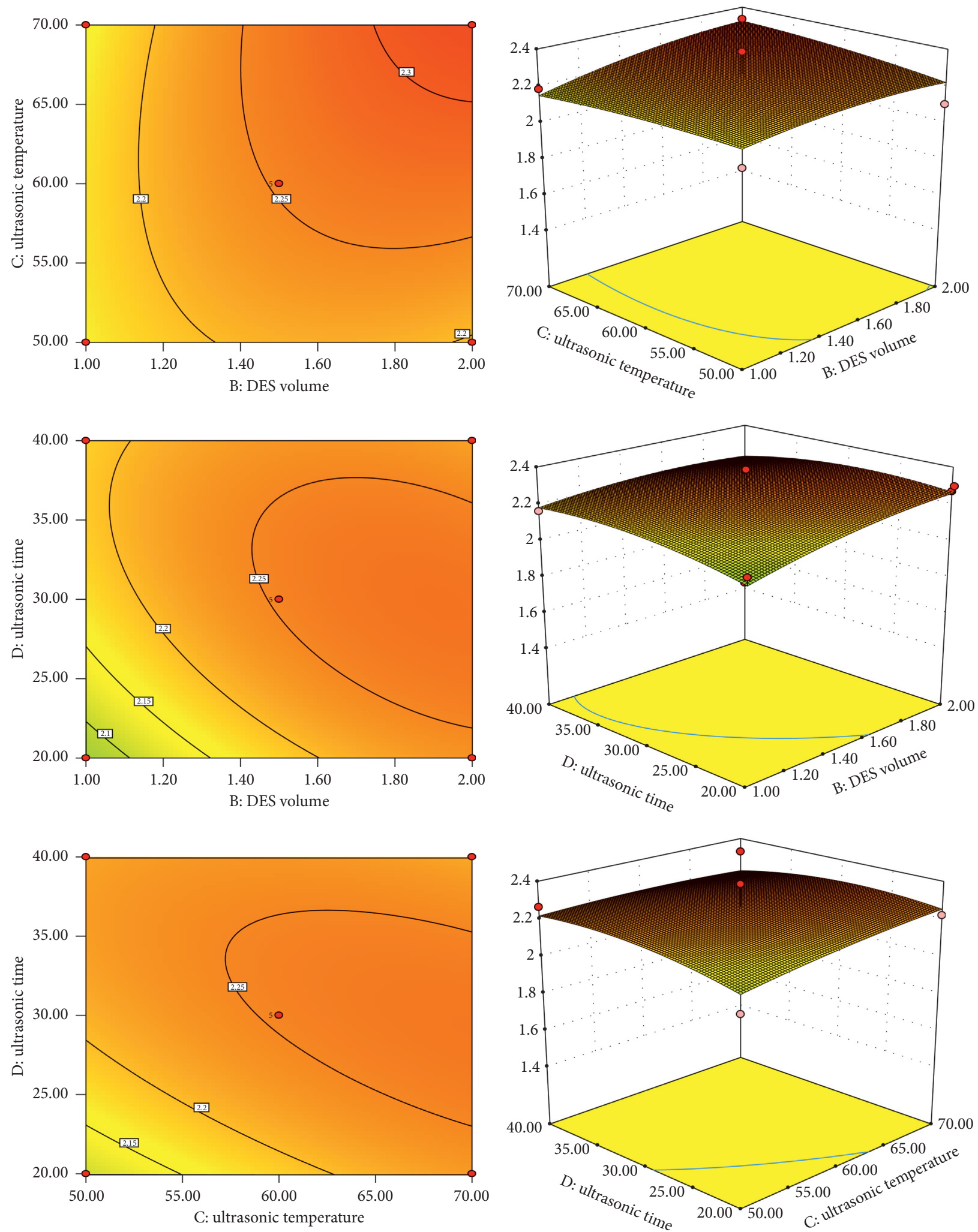

(b)

FIGURE 9: Response surface drawings of CI value analyzed by interaction of two factors. 


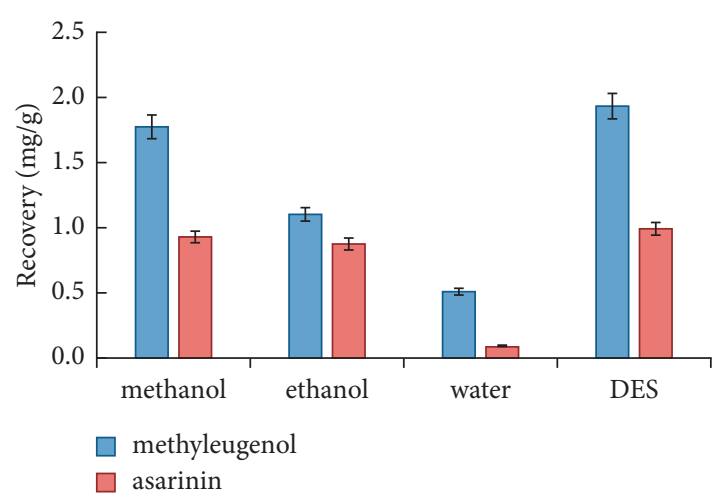

Figure 10: Comparison of other extraction solvents.

3.7. Effect of Vortex Time. In the extraction of sample, the vortex oscillation is mainly to ensure that the sample and the extraction solvent can be fully mixed to form a uniform solvent system so that the target components can be completely extracted. Therefore, in the study, the changes of extraction rate during the eddy time in the range of 3-9 min were investigated. As shown in Figure 8, with the longer vortex time, the extraction rates of methyl eugenol and asarinin increased slightly, but after more than $7 \mathrm{~min}$, almost no change was observed in the extraction rates, indicating that the optimal extraction rate could be achieved when the vortex time was 7 min. Therefore, the time of the first eddy oscillation was finally determined to be $7 \mathrm{~min}$.

\subsection{Optimization of Box-Behnken Response Surface Experiment}

3.8.1. Results of Response Surface Experiment. The response surface test design and results are shown in Table 5.

3.8.2. Model Establishment and Analysis. The analysis of experimental data was performed by Design-Expert 8.0.6 software. The results were fitted by multiple regression, and the regression equation $Y=2.25+0.16 \mathrm{~A}+0.051 \mathrm{~B}$ $+0.029 \mathrm{C}+0.024 \mathrm{D}+0.17 \quad \mathrm{AB}-0.027 \mathrm{AC}-0.029 \mathrm{AD}+$ $0.033 \mathrm{BC}-0.033 \mathrm{BD}-0.031 \mathrm{CD}-0.52 \mathrm{~A}^{2}-0.032 \mathrm{~B}^{2}-0.015 \mathrm{C}^{2}$ $-0.045 \mathrm{D}^{2}$ was obtained, with $R^{2}=0.9429$, indicating that the model can be used for analysis and prediction due to its good fitting and few experimental errors. Further analysis of variance was performed on the equation, and the results are shown in Table 6.

According to the results in Table 3, for the comprehensive evaluation value $\mathrm{CI}, A$ and $A^{2}$ factors all reached extremely significant levels $(P<0.01)$. The influence of the interaction term $\mathrm{AB}$ was statistically significant $(P<0.05)$. There was no statistically significant difference found in the influence of misfitting terms, suggesting that the regression equation presented a good fitting. The overall regression model developed reaches the extremely significant level $(P<0.01)$. The order of the influence of each factor on CI value is $A>B>C>D$.
3.8.3. Interaction of Various Factors. The response surface diagram of the quadratic regression equation was obtained by Design-Expert 8.0.6 software, and the shape of the response surface diagram was investigated. The effects of molar ratio, DES volume, ultrasonic temperature, and ultrasonic time on the extraction rate of methyl eugenol and asarinin were analyzed. The response surface diagram and contour line can well reflect the interaction between the independent variables. By observing the slope of the response surface diagram, the influence of two factors on the response value can be determined. The steeper the response surface diagram is, the more obvious the interaction between the two factors is. As shown in Figure 9, the surface slope of $A B$ 's interaction is relative steep, indicating that the combination of molar ratio and DES volume has a great influence on the extraction rate of methyl eugenol and asarinin in Asarum.

3.8.4. Optimization of Extraction Conditions and Model Verification Results. The optimum extraction conditions of methyl eugenol and asarinin in Asarum obtained by the response surface method were as follows: molar ratio of 1 : 3.3, DES volume of $2 \mathrm{~mL}$, ultrasonic temperature of $70 \mathrm{C}$, ultrasonic time of $24.61 \mathrm{~min}$, and the theoretical comprehensive index (CI) was 2.3736. Combined with the actual needs, the optimum process conditions are modified: molar ratio was $1: 3$, DES volume was $2 \mathrm{~mL}$, ultrasonic temperature was $70^{\circ} \mathrm{C}$, and ultrasonic time was $25 \mathrm{~min}$. Under these conditions, by conducting parallel experiment 3 times, the contents of methyl eugenol and asarinin was $1.9428 \mathrm{mg} / \mathrm{g}$ and $0.9989 \mathrm{mg} / \mathrm{g}$, respectively, and the CI was 2.3280 (RSD of $1.91 \%)$. It was 0.046 lower than the model's CI, which was related to the actual correction molar ratio, and the ultrasound time because both had a significant impact on the results, which shows that the prediction of the model established by Design-Expert 8.0.6 has a good accuracy. As a consequence, the technological conditions of methyl eugenol and asarinin in Asarum DES extraction can be well optimized.

3.9. Comparison with Other Extraction Solvents. The results of the DES extraction were compared with those of common solvent extraction, such as water extraction, methanol extraction, and ethanol extraction. In terms of the experimental process, the DES extraction is simple and stable and does not require a large number of reagents. Furthermore, the extraction time is significantly shortened and the process of solvent concentration is removed [23, 24]. As can be seen from Figure 10, the extraction effect of the DES is better than that of conventional solvent extraction.

\section{Conclusion}

In this paper, a green, efficient, and economical novel extraction method was put forward $[25,26]$. The effects of 13 solvents including water, ethanol, methanol, and 10 kinds of DESs on the extraction rates of asarinin and methyl eugenol were compared. Through single-factor experiments and 
response surface test, the optimal extraction process conditions of asarinin and methyl eugenol in Asarum were obtained: choline chloride and glycerol as the extraction solvent, with a molar ratio of $1: 3$, a DES volume of $2 \mathrm{ml}$, an ultrasonic temperature of $60^{\circ} \mathrm{C}$, a ultrasonic time of $30 \mathrm{~min}$, and a vortex oscillation of $7 \mathrm{~min}$. Under the optimal extraction conditions, the extraction rates of methyl eugenol and asarinin were $1.9428 \mathrm{mg} / \mathrm{g}$ and $0.9989 \mathrm{mg} / \mathrm{g}$. The extraction conditions of this method were mild and did not require high temperature and high pressure and with higher extraction rate and lower loss rates from hydrolysis and biodecomposition. On top of that, the DES is green, natural, nontoxic, and nonpolluting [27, 28], and thus, the extract can be applied to food, medicine, and other fields.

\section{Data Availability}

The main table and figure data used to support the findings of this study are included within the article.

\section{Conflicts of Interest}

The authors declare that they have no conflicts of interest.

\section{Acknowledgments}

This work was supported by a grant from the National Key Research and Development Program of China: "Demonstration research on the standardized cultivation of highquality genuine herbs such as Radix Saposhnikoviae, Schisandra chinensis and Asarum" (2019YFC1710700).

\section{References}

[1] National Pharmacopoeia Commission, Pharmacopoeia in the People's Republic of China: One, pp. 240-241, China Pharmaceutical Technology Press, Beijing, China, 2020.

[2] Yu. Huang, Effect of the Content Determination of Finely Hydrolysis/Efficiency Ingredients and the Impact of the Antidrug/Efficiency Component, Chengdu University of Traditional Chinese Medicine, Chengdu, China, 2013.

[3] Y. Fu, Y. Wang, and R. Zhang, "Research progress on extraction methods and pharmacological activities of asarum," Special Wild Economic Animal and Plant Research, vol. 42, no. 6, pp. 85-89+95, 2020.

[4] Y. Wang, W. Huang, and R. Sun, "Research development on chemical compositions of asarum based on efficacy and toxicity," Chinese Journal of Pharmacovigilance, vol. 10, no. 1, pp. 36-38, 2013.

[5] W. Qingchun and H. Lv, "Separation and identification of Skylin chemical composition in Seoul," Lishizhen Medicine and Materia Medica Research, vol. 21, no. 3, pp. 676-677, 2010.

[6] M. Zhu, Separation and Extraction and Properties of Esotycalized Sugar, Changchun: Northeast Normal University, Changchun, China, 2007.

[7] J. Li and X. Ni, "Separation and purification and immunological activity of esotycalized sugar," Journal of Changchun Normal University: Science edition, vol. 27, no. 1, pp. 54-58, 2008.

[8] J. Lin, H. Liu, D. Sha, Q. Ding, and M. Jiang, "Exploration about the clinical application and dosage of wildginger," Jilin Journal of Chinese Medicine, vol. 41, no. 2, pp. 259-263, 2021.
[9] Y. Zhu, X. Hou, and F. Zhao, "Research progress of asarum application security use," Chinese Archives of Traditional Chinese Medicine, vol. 28, no. 6, pp. 1175-1177, 2010.

[10] P. Abbott Andrew, C. Glen, L. Davies David, K. Rasheed Raymond, and T. Vasuki, "Novel solvent properties of choline chloride/urea mixtures," Chemical Communications, vol. 1, pp. 1-70, 2003.

[11] Q. Zhang, K. De Oliveira Vigier, S. Royer, and F. Jérôme, "Deep eutectic solvents: syntheses, properties and applications," Chemical Society Reviews, vol. 41, no. 21, 2012.

[12] B. Sylwia and A. Jakub, "Evaluation of new natural deep eutectic solvents for the extraction of isoflavones from soy products," Talanta, vol. 168, 2017.

[13] X.-H. Wang and J.-P. Wang, "Effective extraction with deep eutectic solvents and enrichment by macroporous adsorption resin of flavonoids from Carthamus tinctorius L.," Journal of Pharmaceutical and Biomedical Analysis, vol. 176, 2019.

[14] X. S. Yin, Z. F. Zhong, G. L. Bian, X. J. Cheng, and D. Q. Li, "Ultra-rapid, enhanced and eco-friendly extraction of four main flavonoids from the seeds of Oroxylum indicum by deep eutectic solvents combined with tissue-smashing extraction," Food Chemistry, vol. 319, 2019.

[15] D. Chandran, M. Khalid, R. Walvekar et al., "Deep eutectic solvents for extraction-desulphurization: a review," Journal of Molecular Liquids, vol. 275, 2019.

[16] A. Satlewal, R. Agrawal, S. Bhagia, P. Das, and A. J. Ragauskas, "Rice straw as a feedstock for biofuels: availability, recalcitrance, and chemical properties," Biofuels, Bioproducts and Biorefining, vol. 12, no. 1, 2018.

[17] L. Capolupo and V. Faraco, "Green methods of lignocellulose pretreatment for biorefinery development," Applied Microbiology and Biotechnology, vol. 100, no. 22, 2016.

[18] K. Teng, P. Shen, J. Sun, and H. Zhang, "Optimization of Ultrasonic-assisted Extraction of Antioxidant Compounds from Asari Radix et Rhizoma Using Response Surface Methodology," Chinese Pharmaceutical Journal, vol. 55, no. 18, pp. 1499-1504, 2020.

[19] M. Ramdin, T. W. de Loos, and T. J. H. Vlugt, "State-of-the-Art of $\mathrm{CO} 2$ capture with ionic liquids," Industrial and Engineering Chemistry Research, vol. 51, no. 24, pp. 8149-8177, 2012.

[20] Z. Li, Y. Ha, A. Li, W. Li, F. Wang, and L. Qing-Peng, "Study on extraction process of apple slag polyphenol ultrasound extraction," Scientia Agricultura Sinica, vol. 46, no. 21, pp. 4569-4577, 2013.

[21] R. F. Contamine, A. M. Wilhelm, J. Berlan, and H. Delmas, "Power measurement in sonochemistry," Ultrasonics-Sonochemistry, vol. 2, no. 1, 1995.

[22] B. Wang, X. Zhang, H. Pan, D. Lv, Y. Fan, and H. Liu, "Response surface method optimizes purslane flavonoid ultrasonic extraction process," Jiangsu Journal of Agricultural Sciences, vol. 34, no. 1, pp. 166-171, 2018.

[23] Z. Zhao, Y. Ji, X. Liu, and L. Zhao, "Research progress of extraction separation technology and its application based on low eutectic solvent," Chinese Journal of Chromatography, vol. 39, no. 2, pp. 152-161, 2021.

[24] O.-Y. Tianzhi and Q. Yuan, "Optimization of extraction process of methyl eugenol from volatile oil of asarum Sieboldii Miq. by ultrasound-assisted extraction," Natural Product Research and Development, vol. 25, no. 02, pp. 224-228+240, 2013.

[25] Z. Fang and L. Xing, "Deep eutectic solvent technology and its application," Shandong Chemical Industry, vol. 49, no. 24, pp. 136-138, 2020. 
[26] L. Li, Y. Wang, F. Liu, Y. Xu, and H. Bao, "Study on the effect of deep eutectic solvent liquid phase microextraction on quality standard, antitussive, and expectorant of sangbaipi decoction," Journal of Analytical Methods in Chemistry, vol. 2021, Article ID 9999406, 11 pages, 2021.

[27] X. Yue, F. Xu, P. Lv, H. Yang, H. Bao, and Y. Xu, "Analysis of antioxidant capacity of chromones in saposhnikoviae radix obtained by ultrasonic-assisted deep eutectic solvents extraction," Journal of Analytical Methods in Chemistry, vol. 2020, Article ID 8875788, 9 pages, 2020.

[28] S. Chen, L. Liu, L. Zhao, and L. Zhu, "Ultrasound auxiliary deep eutectic solvent method to extract the residual pesticide," Food Research and Development, vol. 41, no. 3, pp. 202-207, 2020 . 